Available Online : https://proceeding.researchsynergypress.com/index.php/cset/index

RSF Conference Series: Engineering and Technology

ISSN 2809-6843 (Online) | 2809-6878 (Print)

Volume 1 Number 1 (2021): 407-413

\title{
The Comparison of Tree-Based Ensemble Machine Learning for Classifying Public Datasets
}

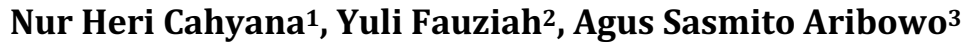 \\ 1, 2, 3Informatics Department, Universitas Pembangunan Nasional “Veteran” Yogyakarta, Indonesia
}

\begin{abstract}
This study aims to determine the best methods of tree-based ensemble machine learning to classify the datasets used, a total of 34 datasets. This study also wants to know the relationship between the number of records and columns of the test dataset with the number of estimators (trees) for each ensemble model, namely Random Forest, Extra Tree Classifier, AdaBoost, and Gradient Bosting. The four methods will be compared to the maximum accuracy and the number of estimators when tested to classify the test dataset. Based on the results of the experiments above, tree-based ensemble machine learning methods have been obtained and the best number of estimators for the classification of each dataset used in the study. The Extra Tree method is the best classifier method for binary-class and multi-class. Random Forest is good for multi-classes, and AdaBoost is a pretty good method for binary-classes. The number of rows, columns and data classes is positively correlated with the number of estimators. This means that to process a dataset with a large row, column or class size requires more estimators than processing a dataset with a small row, column or class size. However, the relationship between the number of classes and accuracy is negatively correlated, meaning that the accuracy will decrease if there are more classes for classification.
\end{abstract}

Keywords: Tree-Based Ensemble Machine Learning, Public Datasets, Estimators, Accuracy

This is an open access article under the CC-BY-NC license

\section{INTRODUCTION}

Classification is an important part of data mining to solve business and technical problems. Classification using machine learning is supervised. This algorithm requires knowledgeable data to build a model. One measure of the success of the classification model is the accuracy of the model. The accuracy of the model depends on several things, ranging from the condition of the dataset, preprocessing techniques, feature extraction methods, and machine learning algorithms. The characteristic of supervised learning is a learning method that reuses data and outputs that have been entered by users or done by the system in the past. Some examples of basic classification algorithms that apply supervised learning methods are the Naïve Bayes algorithm, Decision Tree, Support Vector Machine, and K-Nearest Neighbour.

Many researches on machine learning algorithms for public dataset classification have been carried out, including the iris dataset (Vatshayan, 2019). These basic algorithms have their characteristic limitations. Especially for tree-based methods, its limitations are sensitive to outliers, unstable and allow overfitting to occur. The development of a tree-based algorithm is an ensemble tree. Tree based ensemble machine learning are machine learning methods that apply bagging and boosting techniques using tree-based algorithms. Bagging is a method that can improve the results of machine learning classification algorithms by combining prediction classifications from several models. It is used to overcome instability in complex models with relatively small data sets. Bagging is one of the earliest and simplest and most effective ensemble- based algorithms. Bagging is best suited for problems with relatively small training datasets. Bagging adopts bootstrap distribution in order to generate different base learners, to obtain subset data. bagging also adopts a base learner output aggregation strategy, namely the voting method for classification cases and averaging for regression cases. The method uses multiple versions of a training set by using the bootstrap, i.e. sampling with replacement (Sewell, 2009). 
Boosting is a way to generate multiple models or classifiers for prediction or classification, and also combine predictions from multiple models into a single prediction. Boosting is an iterative approach to generate strong classifiers and minimal training errors from a group of weak classifiers. Boosting is generally designed for binary class problems.

Research related to the use of ensemble tree-based algorithms is (Raghavendra \& Santosh Kumar, 2020) about the performance of random forest in prediction of diabetes on Pima Indian dataset. The experiment was conducted using R studio platform and achieved classification accuracy of $84.1 \%$. Another research is (Rajendar et al., 2020) on classifier models for early prediction of diabetes. In this research, random forest gives accuracy 78.6\%. Research from (Yu et al., 2020) used five datasets including Blood, Haberman, Iris, Seeds and Wine from the UCI dataset and used modified random forest for machine learning. The result of this research is the classification accuracy of each dataset, namely Blood (75.94\%), Haberman (72.37\%), Iris (73.90\%), Seeds (71.15\%), and Wine $(75.64 \%)$. Research from (Sharma et al., 2020) used the iris dataset to be classified by Random Forest with very satisfactory results. Research from (Cahyana et al., 2019) uses seven datasets to be classified using Gradient Boosting. The results of the classification accuracy of each dataset are for Mammography (0.78), Liver Disorders (0.77), Pima Indian (0.79), Indian Liver (0.71), Haberman (0.62), and Immunotherapy $(0,83)$. This study succeeded in increasing the accuracy of around $2-11 \%$ after oversampling. Research (Prasetiyowati et al., 2020) describes the performance of the Random Forest method on high-dimensional datasets such as the Parkinson, CNAE-9, and Urban Land Cover datasets. The average accuracy using K-fold Cross Validation for Parkinson (86.66\%), CNAE-9 (93.72\%), and Urban Land Cover (85.08\%). The study (Ahmad et al., 2021) used HbA1c and FPG labelled datasets and the accuracy for HbA1c (81.48\%) and FPG (88.27\%). The studies above do not explain how many best estimators (trees) are used to produce high accuracy. This knowledge is needed to determine the complexity of the machine learning process which can ultimately be useful for considering the treebased ensemble machine learning method to be chosen.

This study aims to determine the best methods of tree-based ensemble machine learning to classify the datasets used, a total of 34 datasets. This study also wants to know the relationship between the number of records and columns of the test dataset with the number of estimators (trees) for each ensemble model. There are 4 tree-based ensemble machine learning methods to be compared in this study, namely Random Forest, Extra Tree Classifier, AdaBoost, and Gradient Bosting. The four methods will be compared to the maximum accuracy and the number of estimators when tested to classify the test dataset. Specifically, the Random Forest and Extra Tree Classifier methods have been studied before, for example for sentiment analysis (A S Aribowo et al., 2020) and cross domain sentiment analysis (Agus Sasmito Aribowo et al., 2021). To measure maximum accuracy, this study will try out estimator values from the lowest to the highest on the four tree-based ensemble machine learning methods.

\section{RESEARCH METHOD}

This research is an experimental study to determine the best tree-based ensemble machine learning method for several public datasets. The research starts from getting the dataset, cleaning the dataset, eliminating the damaged data, and imputing the missing data.

The datasets used in this study are public datasets available in several repositories such as the UCI Machine Learning Repository (https://archive.ics.uci.edu/), datahub.io, and several other sites that allow the dataset to be freely downloaded. . We conducted trials on 34 types of datasets that varied in the number of records, the number of columns, and classes. Our datasets are divided into two groups, namely binary-class and multi-class datasets as shown in Table 1. 
RSF RSF Conference Series: Engineering and Technology

Vol 1 (1), 407-413

The Comparison of Tree-Based Ensemble Machine Learning for Classifying Public Datasets

Nur Heri Cahyana, Yuli Fauziah, Agus SasmitoAribowo

Table 1. Datasets for Experiment

\begin{tabular}{|l|l|r|r|r|}
\hline \multicolumn{3}{|l|}{ Binary-Class } \\
\hline No. & Dataset & $\begin{array}{l}\text { Record } \\
\text { Number }\end{array}$ & $\begin{array}{l}\text { Column } \\
\text { Number }\end{array}$ & $\begin{array}{l}\text { llass } \\
\text { Number }\end{array}$ \\
\hline 1 & Sonar & 208 & 61 & 2 \\
\hline 2 & WDBC & 360 & 31 & 2 \\
\hline 3 & Iono & 351 & 35 & 2 \\
\hline 4 & WPBC & 194 & 34 & 2 \\
\hline 5 & Haber & 306 & 4 & 2 \\
\hline 6 & Musk1 & 476 & 167 & 2 \\
\hline 7 & Musk2 & 6598 & 167 & 2 \\
\hline 8 & PIMA & 768 & 9 & 2 \\
\hline 9 & Park & 195 & 23 & 2 \\
\hline 10 & Climate & 540 & 21 & 2 \\
\hline 11 & Trans & 748 & 5 & 2 \\
\hline 12 & plrx & 182 & 13 & 2 \\
\hline 13 & QSAR & 1055 & 42 & 2 \\
\hline 14 & Bank & 1372 & 5 & 2 \\
\hline 15 & Hill & 1212 & 101 & 2 \\
\hline 16 & Thyroid & 2000 & 25 & 2 \\
\hline
\end{tabular}

\begin{tabular}{|l|l|r|r|r|}
\hline \multicolumn{3}{|l|}{ Multi-Class } \\
\hline No. & Dataset & $\begin{array}{l}\text { Record } \\
\text { Number }\end{array}$ & $\begin{array}{l}\text { Column } \\
\text { Number }\end{array}$ & $\begin{array}{l}\text { Class } \\
\text { Number }\end{array}$ \\
\hline 1 & Seeds & 210 & 8 & 3 \\
\hline 2 & Wine & 178 & 14 & 3 \\
\hline 3 & Iris & 150 & 6 & 3 \\
\hline 4 & Glass & 214 & 11 & 6 \\
\hline 5 & Libras & 360 & 91 & 15 \\
\hline 6 & CNAE & 1080 & 857 & 9 \\
\hline 7 & Vowel & 990 & 14 & 10 \\
\hline 8 & DUser & 258 & 6 & 4 \\
\hline 9 & Ecoli & 307 & 9 & 4 \\
\hline 10 & Letter & 20000 & 17 & 26 \\
\hline 11 & Robot & 5456 & 5 & 4 \\
\hline 12 & Cardio & 2126 & 23 & 10 \\
\hline 13 & Opt & 5620 & 65 & 10 \\
\hline 14 & Landsat & 6435 & 37 & 6 \\
\hline 15 & Page & 5473 & 10 & 5 \\
\hline 16 & Pen & 10992 & 17 & 10 \\
\hline 17 & CNAE & 1080 & 857 & 9 \\
\hline 18 & WRed & 1599 & 12 & 6 \\
\hline
\end{tabular}

The research will try out four tree-based ensemble machine learning on all datasets in this study. Each model will also be tested to find out how many bootstraps (trees) are optimally providing the highest accuracy. So we created a number of models for each bootstrap test. The number of bootstrap is also known as the number of estimators. The number of estimators will be tried starting from 10 estimators, 20 estimators, and so on up to 200 estimators. More details can be found in the research steps.

\section{Research steps}

The research steps are the sequences of the research process to answer the research questions. The question is what is the best tree-based ensemble machine learning method for each dataset and how many estimators to achieve it. The unit of measurement is classification accuracy. All datasets are used in research and get trials from all tree-based ensemble machine learning with the number of each estimator varying between 10 estimators to 200 estimators.

The research steps were :

1. Normalize data in every dataset. Handling missing values using imputation methods.

2. READ dataset $\mathrm{X}$

a. Split dataset into data training and data testing with ratio 8:2

b. Set estimator, $\mathrm{N}=10 . .200$, STEP 10 :

i. Creating 4 models using ensemble machine learning and data training

1. Model 1: Using Random Forest(estimator $=\mathrm{N}$ )

2. Model 2: Using AdaBoost(estimator $=\mathrm{N}$ )

3. Model 3: Using Gradient Boosting(estimator $=\mathrm{N}$ )

4. Model 4: Using Extra Tree Classifier(estimator $=\mathrm{N}$ )

ii. Validate data testing using Model 1, Model2, Model 3 and Model4.

c. Get Max(Accuracy) and N from Validation Model1, Model2, Model3, and Model4. 
RSF Conference Series: Engineering and Technology

Vol 1 (1), 407-413

The Comparison of Tree-Based Ensemble Machine Learning for Classifying Public Datasets

Nur Heri Cahyana, Yuli Fauziah, Agus Sasmito Aribowo

The results of the research are the best accuracy figures from each tree-based ensemble machine learning in each dataset. Another research result is the number of estimators to get the highest accuracy.

\section{RESULTS}

The result of the research is the maximum accuracy rate obtained for each type of tree-based ensemble machine learning ensemble per dataset after trying estimators ranging from 10, 20, to 200 . The output of each experiment is the maximum accuracy and the estimator for each type of tree-based ensemble machine learning per dataset. Table 2 is a summary of the experimental results for all binary-class datasets. Table 3 is a summary of the experimental results for all multi-class datasets.

Table 2. Summary of The Experimental Results for All Binary-Class Datasets

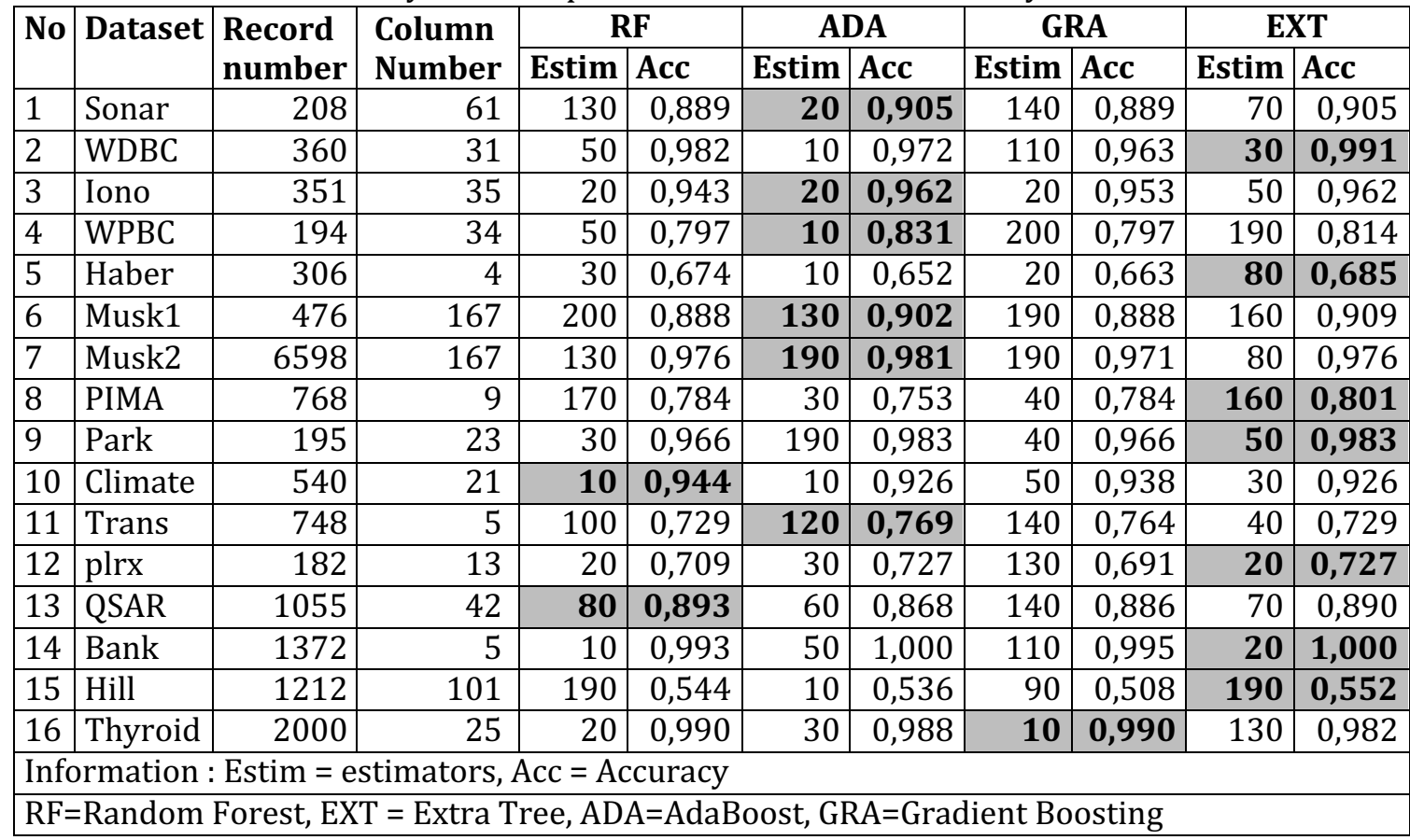

Table 2 shows that the best accuracy for the classification of binary-class datasets is the extra tree classifier, followed by AdaBoost. Extra Tree classifier is very good at classifying Bank, WDBC and Park datasets. While AdaBoost is better at classifying Sonar, Iono, WPBC, and Musk1 datasets. This assessment is carried out by observing the accuracy value and the number of estimators. The fewer estimators, the algorithm works more efficiently and processing time will certainly be faster. Table 2 also shows that there is a relationship between the number of dataset records and the number of estimators.

Table 3. Summary of the Experimental Results for All Multi-Class Datasets

\begin{tabular}{|c|c|c|c|c|c|c|c|c|c|c|c|c|}
\hline \multirow{2}{*}{\begin{tabular}{|l|}
$\mathbf{N}$ \\
$\mathbf{0}$
\end{tabular}} & \multirow{2}{*}{$\begin{array}{l}\text { Datase } \\
\mathrm{t}\end{array}$} & \multirow{2}{*}{$\begin{array}{l}\text { Rec } \\
\text { Num }\end{array}$} & \multirow{2}{*}{$\begin{array}{l}\text { Column } \\
\text { Numbe } \\
r\end{array}$} & \multirow{2}{*}{\begin{tabular}{l|} 
Clas \\
s \\
Num
\end{tabular}} & \multicolumn{2}{|l|}{ RF } & \multicolumn{2}{|l|}{ ADA } & \multicolumn{2}{|l|}{ GRA } & \multicolumn{2}{|l|}{ EXT } \\
\hline & & & & & $\begin{array}{l}\text { Esti } \\
\text { m }\end{array}$ & Acc & $\begin{array}{l}\text { Esti } \\
\text { m }\end{array}$ & Acc & $\begin{array}{l}\text { Esti } \\
\text { m }\end{array}$ & Acc & \begin{tabular}{|l|} 
Esti \\
$\mathbf{m}$
\end{tabular} & Acc \\
\hline 1 & Seeds & 210 & 8 & 3 & 10 & $\begin{array}{r}0.95 \\
2 \\
\end{array}$ & 30 & $\begin{array}{r}0.93 \\
7 \\
\end{array}$ & 110 & 0.952 & 10 & $\begin{array}{r}0.95 \\
2 \\
\end{array}$ \\
\hline 2 & Wine & 178 & 14 & 3 & 40 & 1.000 & 10 & $\begin{array}{r}0.92 \\
6 \\
\end{array}$ & 20 & 0.963 & 30 & $\begin{array}{r}1.00 \\
0 \\
\end{array}$ \\
\hline 3 & Iris & 150 & 6 & 3 & 10 & $\begin{array}{r}1.00 \\
0\end{array}$ & 10 & $\begin{array}{r}0.97 \\
8 \\
\end{array}$ & 10 & 0.978 & 10 & $\begin{array}{r}1.00 \\
0 \\
\end{array}$ \\
\hline 4 & Glass & 214 & 11 & 6 & 20 & 0.985 & 10 & $\begin{array}{r}0.83 \\
1\end{array}$ & 10 & 0.985 & 30 & $\begin{array}{r}1.00 \\
0\end{array}$ \\
\hline
\end{tabular}


RSF RSF Conference Series: Engineering and Technology

Vol 1 (1), 407-413

The Comparison of Tree-Based Ensemble Machine Learning for Classifying Public Datasets

Nur Heri Cahyana, Yuli Fauziah, Aqus Sasmito Aribowo

\begin{tabular}{|c|c|c|c|c|c|c|c|c|c|c|c|c|}
\hline 5 & Libras & 360 & 91 & 15 & 190 & 0.872 & 10 & $\begin{array}{r}0.10 \\
1\end{array}$ & 30 & 0.752 & 60 & $\begin{array}{r}0.89 \\
9\end{array}$ \\
\hline 6 & CNAE & 1080 & 857 & 9 & 60 & 0.914 & 10 & $\begin{array}{r}0.53 \\
1\end{array}$ & 70 & 0.907 & 60 & $\begin{array}{r}0.92 \\
6\end{array}$ \\
\hline 7 & Vowel & 990 & 14 & 10 & 140 & 0.973 & 70 & $\begin{array}{r}0.18 \\
2 \\
\end{array}$ & 190 & 0.906 & 40 & \begin{tabular}{r|}
0.99 \\
0
\end{tabular} \\
\hline 8 & DUser & 258 & 6 & 4 & 40 & 0.962 & 10 & $\begin{array}{r}0.80 \\
8\end{array}$ & 180 & 0.949 & 70 & $\begin{array}{r}0.97 \\
4\end{array}$ \\
\hline 9 & Ecoli & 307 & 9 & 4 & 30 & 0.978 & 10 & $\begin{array}{r}0.83 \\
9\end{array}$ & 20 & $\begin{array}{r}0.98 \\
9\end{array}$ & 110 & 0.978 \\
\hline 10 & Letter & $\begin{array}{r}2000 \\
0\end{array}$ & 17 & 26 & 150 & 0.962 & 20 & $\begin{array}{r}0.32 \\
9\end{array}$ & 200 & 0.946 & 180 & 0.971 \\
\hline 11 & Robot & 5456 & 5 & 4 & 10 & $\begin{array}{r}1.00 \\
0\end{array}$ & 10 & $\begin{array}{r}0.85 \\
0\end{array}$ & 180 & 0.999 & 80 & 0.995 \\
\hline 12 & Cardio & 2126 & 23 & 10 & 30 & $\begin{array}{r}0.91 \\
2\end{array}$ & 10 & $\begin{array}{r}0.48 \\
4\end{array}$ & 130 & 0.906 & 80 & 0.908 \\
\hline 13 & Opt & 5620 & 65 & 10 & 130 & 0.988 & 20 & $\begin{array}{r}0.67 \\
5\end{array}$ & 180 & 0.979 & 140 & $\begin{array}{r}0.99 \\
0\end{array}$ \\
\hline 14 & Landsat & 6435 & 37 & 6 & 130 & 0.919 & 30 & $\begin{array}{r}0.77 \\
5\end{array}$ & 200 & 0.909 & 110 & 0.91 \\
\hline 15 & Page & 5473 & 10 & 5 & 70 & $\begin{array}{r}0.97 \\
1\end{array}$ & 10 & $\begin{array}{r}0.92 \\
0\end{array}$ & 190 & 0.968 & 30 & 0.970 \\
\hline 16 & Pen & $\begin{array}{r}1099 \\
2\end{array}$ & 17 & 10 & 180 & 0.993 & 40 & $\begin{array}{r}0.28 \\
1\end{array}$ & 160 & 0.991 & 40 & 0.99 \\
\hline 17 & CNAE & 1080 & 857 & 2 & 90 & 0.914 & 10 & $\begin{array}{r}0.53 \\
1\end{array}$ & 50 & 0.907 & 140 & $\begin{array}{r}0.93 \\
2\end{array}$ \\
\hline 18 & WRed & 1599 & 12 & 9 & 190 & 0.688 & 10 & $\begin{array}{r}0.59 \\
2\end{array}$ & 120 & 0.650 & 120 & $\begin{array}{r}0.69 \\
6\end{array}$ \\
\hline
\end{tabular}

Table 3 shows that the best accuracy for the classification of multiclass datasets is extra tree classifier, followed by Random Forest. Extra Tree classifier is very good at classifying almost all datasets. Meanwhile, Random Forest is very good for classifying Robot and Cardio datasets. This assessment is carried out by observing the accuracy value and the number of estimators. Likewise in experiments on binary-class datasets, the fewer the number of estimators, the more efficient the algorithm works and the faster processing time. Table 3 also shows that there is a relationship between the number of dataset records and the number of estimators.

Then it will be calculated how much is the relationship between the number of records and the number of the best method estimators for binary-class and multi class. Table 4 shows the relationship between the number of dataset records and the number of estimators in the binary-class datasets.

Table 5. Relationship between the number of records, columns and the estimator count in multi-class datasets.

Table 4. Relationship between the Number of Dataset Records, Column and Number of Estimators in the Binary-Class Datasets

\begin{tabular}{|l|l|r|r|r|r|l|}
\hline No & Dataset & $\begin{array}{l}\text { Record } \\
\text { number }\end{array}$ & $\begin{array}{l}\text { Column } \\
\text { Number }\end{array}$ & $\begin{array}{l}\text { Estimator } \\
\text { Number }\end{array}$ & Best Accuracy & Best Method \\
\hline 1 & Sonar & 208 & 61 & 20 & 0,905 & ADA \\
\hline 2 & WDBC & 360 & 31 & 30 & 0,991 & EXT \\
\hline 3 & Iono & 351 & 35 & 20 & 0,962 & ADA \\
\hline
\end{tabular}


RSF Conference Series: Engineering and Technology

Vol 1 (1), 407-413

The Comparison of Tree-Based Ensemble Machine Learning for Classifying Public Datasets

Nur Heri Cahyana, Yuli Fauziah, Aqus Sasmito Aribowo

\begin{tabular}{|l|l|r|r|r|r|l|}
\hline 4 & WPBC & 194 & 34 & 10 & 0,831 & ADA \\
\hline 5 & Haber & 306 & 4 & 80 & 0,685 & EXT \\
\hline 6 & Musk1 & 476 & 167 & 130 & 0,902 & ADA \\
\hline 7 & Musk2 & 6598 & 167 & 190 & 0,981 & ADA \\
\hline 8 & PIMA & 768 & 9 & 160 & 0,801 & EXT \\
\hline 9 & Park & 195 & 23 & 50 & 0,983 & EXT \\
\hline 10 & Climate & 540 & 21 & 10 & 0,944 & RF \\
\hline 11 & Trans & 748 & 5 & 120 & 0,769 & ADA \\
\hline 12 & plrx & 182 & 13 & 20 & 0,727 & EXT \\
\hline 13 & QSAR & 1055 & 42 & 80 & 0,893 & RF \\
\hline 14 & Bank & 1372 & 5 & 20 & 1,0 & EXT \\
\hline 15 & Hill & 1212 & 101 & 190 & 0,552 & EXT \\
\hline 16 & Thyroid & 2000 & 25 & 10 & 0,990 & GRA \\
\hline \multicolumn{7}{|l}{ Method Information : } \\
\hline RF=Random Forest, EXT = Extra Tree, ADA=AdaBoost, GRA=Gradient Boosting \\
\hline
\end{tabular}

If it is calculated using Pearson correlation, the variable number of rows and the estimator is correlated with a score of 0.497 , where the Pearson correlation coefficient ( $\mathrm{r}$ ) is a value ranging from -1 to 1 to indicate the strength of the association. This value indicates that the two variables are positively connected. Similarly, the number of columns and the number of estimators are also connected with the Pearson correlation score of 0.579 .

Then it will be calculated how much the relationship between the number of records and the number of the best method estimators for multi-class will be calculated. Table 5 shows the relationship between the number of dataset records and the number of classes in multi-class datasets, with the number of estimators and accuracy values.

Table 5. Relationship between the Number of Dataset Records, Column, Class and Number of Estimators in the Multi-Class Datasets

\begin{tabular}{|l|l|r|r|r|r|l|l|}
\hline No & Dataset & $\begin{array}{l}\text { Record } \\
\text { Number }\end{array}$ & $\begin{array}{l}\text { Column } \\
\text { Number }\end{array}$ & $\begin{array}{l}\text { Class } \\
\text { Number }\end{array}$ & $\begin{array}{l}\text { Estimator } \\
\text { Number }\end{array}$ & $\begin{array}{l}\text { Best } \\
\text { Accuracy }\end{array}$ & $\begin{array}{l}\text { Best } \\
\text { Method }\end{array}$ \\
\hline 1 & Seeds & 210 & 8 & 3 & 10 & 0,952 & EXT \\
\hline 2 & Wine & 178 & 14 & 3 & 30 & 1,000 & EXT \\
\hline 3 & Iris & 150 & 6 & 3 & 10 & 1,000 & EXT \\
\hline 4 & Glass & 214 & 11 & 6 & 30 & 1,000 & EXT \\
\hline 5 & Libras & 360 & 91 & 15 & 60 & 0,899 & EXT \\
\hline 6 & CNAE & 1080 & 857 & 9 & 60 & 0,926 & EXT \\
\hline 7 & Vowel & 990 & 14 & 10 & 40 & 0,990 & EXT \\
\hline 8 & DUser & 258 & 6 & 4 & 70 & 0,974 & EXT \\
\hline 9 & Ecoli & 307 & 9 & 4 & 20 & 0,989 & GRA \\
\hline 10 & Letter & 20000 & 17 & 26 & 180 & 0,971 & EXT \\
\hline 11 & Robot & 5456 & 5 & 4 & 10 & 1,000 & RF \\
\hline 12 & Cardio & 2126 & 23 & 10 & 30 & 0,912 & RF \\
\hline 13 & Opt & 5620 & 65 & 10 & 140 & 0,990 & EXT \\
\hline 14 & Landsat & 6435 & 37 & 6 & 110 & 0,919 & EXT \\
\hline 15 & Page & 5473 & 10 & 5 & 70 & 0,971 & RF \\
\hline
\end{tabular}


RSF RSF Conference Series: Engineering and Technology

Vol 1 (1), 407-413

The Comparison of Tree-Based Ensemble Machine Learning for Classifying Public Datasets

Nur Heri Cahyana, Yuli Fauziah, Agus Sasmito Aribowo

\begin{tabular}{|l|l|r|r|r|r|r|l|}
\hline 16 & Pen & 10992 & 17 & 10 & 40 & 0,995 & EXT \\
\hline 17 & CNAE & 1080 & 857 & 2 & 140 & 0,932 & EXT \\
\hline 18 & WRed & 1599 & 12 & 9 & 120 & 0,696 & EXT \\
\hline Method Information : \\
\hline
\end{tabular}

In Table 5, if calculated using Pearson correlation, the number of rows and estimators is connected to the Pearson correlation score of 0.549 , and the relationship between the number of columns and the estimator variable is connected to the Pearson correlation score of 0.528 . This value indicates that the number of rows and the number of columns is positively related to the number of estimators. The relationship between the number of classes and the number of estimators is also positively correlated with a Pearson correlation score of 0.528 , and the relationship between the number of classes and the negative Pearson correlated accuracy is -0.354 . This means that the number of classes and accuracy will be inversely proportional.

\section{Conclusion}

Based on the results of the experiments above, tree-based ensemble machine learning methods have been obtained and the best number of estimators for the classification of each dataset used in the study. The Extra Tree method is the best classifier method for binary-class and multi-class. Random Forest is good for multi-classes, and AdaBoost is a pretty good method for binary-classes. Then the knowledge is obtained that the number of rows, columns and data classes is positively correlated with the number of estimators. This means that to process a dataset with a large row, column or class size requires more estimators than processing a dataset with a small row, column or class size. However, the relationship between the number of classes and accuracy is negatively correlated, meaning that the accuracy will decrease if there are more classes for classification.

\section{REFERENCES}

Ahmad, H. F., Mukhtar, H., Alaqail, H., Seliaman, M., \& Alhumam, A. (2021). Investigating HealthRelated Features and Their Impact on the Prediction of Diabetes Using Machine Learning. Applied Sciences (Switzerland), 11(3), 1-18. https://doi.org/10.3390/app11031173

Aribowo, A S, Basiron, H., Herman, N. S., \& Khomsah, S. (2020). An evaluation of preprocessing steps and tree-based ensemble machine learning for analysing sentiment on Indonesian youtube comments. International Journal of Advanced Trends in Computer Science and Engineering, 9(5), 7078-7086. https://doi.org/10.30534/ijatcse/2020/29952020

Aribowo, Agus Sasmito, Basiron, H., Yusof, N. F. A., \& Khomsah, S. (2021). Cross-Domain Sentiment Analysis Model On Indonesian Youtube Comment. International Journal of Advances in Intelligent Informatics, 7(1), 12-25. https://doi.org/10.26555/ijain.v7i1.554

Cahyana, N., Khomsah, S., \& Aribowo, A. S. (2019). Improving Imbalanced Dataset Classification Using Oversampling and Gradient Boosting. Proceeding - 2019 5th International Conference on Science in Information Technology: Embracing Industry 4.0: Towards Innovation in Cyber Physical System, ICSITech 2019, 217-222. https://doi.org/10.1109/ICSITech46713.2019.8987499

Prasetiyowati, M. I., Maulidevi, N. U., \& Surendro, K. (2020). Feature selection to increase the random forest method performance on high dimensional data. International Journal of Advances in Intelligent Informatics, 6(3), 303-312. https://doi.org/10.26555/ijain.v6i3.471

Raghavendra, S., \& Santosh Kumar, J. (2020). Performance evaluation of random forest with feature selection methods in prediction of diabetes. International Journal of Electrical and Computer Engineering, 10(1), 353-359. https://doi.org/10.11591/ijece.v10i1.pp353-359

Rajendar, S., Thangaraj, R., Palanisamy, J., \& Kaliappan, V. K. (2020). Comparative Analysis of Classifier 
RSF Conference Series: Engineering and Technology

Vol 1 (1), 407-413

The Comparison of Tree-Based Ensemble Machine Learning for Classifying Public Datasets

Nur Heri Cahyana, Yuli Fauziah, Agus Sasmito Aribowo

Models for the Early Prediction of Type 2 Diabetes. International Journal of Advanced Science and Technolo, 29(7), 2184-2194.

Sewell, M. (2009). Ensemble learning. Academia-UCL Department of Computer Science, 4(1), 2776. https://doi.org/10.4249/scholarpedia.2776

Sharma, K., College, S., \& Rajhasthan. (2020). Classification of IRIS Dataset using Weka. International Journal of Computer Applications \& Information Technology, 12(1), 287-291.

Vatshayan, S. (2019). Performance Evaluation of Supervised Learning for Iris Flower Species. INTERNATIONAL JOURNAL OF INNOVATIVE RESEARCH IN TECHNOLOGY, April.

Yu, Y., Wang, L., Huang, H., \& Yang, W. (2020). An Improved Random Forest Algorithm. Journal of Physics: Conference Series, 1646(1). https://doi.org/10.1088/1742-6596/1646/1/012070

Zhang, Y., \& Haghani, A. (2015). A gradient boosting method to improve travel time prediction. Transportation Research Part C: Emerging Technologies, 58, 308-324. https://doi.org/10.1016/j.trc.2015.02.019 